\title{
Perfect timing: circadian rhythms, sleep, and immunity - an NIH workshop summary
}

Jeffrey A. Haspel, ${ }^{1}$ Ron Anafi, ${ }^{2}$ Marishka K. Brown, ${ }^{3}$ Nicolas Cermakian, ${ }^{4}$ Christopher Depner, ${ }^{5}$ Paula Desplats, ${ }^{6,7}$ Andrew E. Gelman, ${ }^{8}$ Monika Haack, ${ }^{9}$ Sanja Jelic, ${ }^{10}$ Brian S. Kim, ${ }^{11,12,13,14,15}$ Aaron D. Laposky, ${ }^{3}$ Yvonne C. Lee, ${ }^{16}$ Emmanuel Mongodin, ${ }^{17}$ Aric A. Prather, ${ }^{18}$ Brian J. Prendergast, ${ }^{19}$ Colin Reardon, ${ }^{20}$ Albert C. Shaw, ${ }^{21}$ Shaon Sengupta, ${ }^{22,23}$ Éva Szentirmai, ${ }^{24}$ Mahesh Thakkar, ${ }^{25,26}$ Wendy E. Walker, ${ }^{27}$ and Laura A. Solt ${ }^{28}$

'Division of Pulmonary, Critical Care and Sleep Medicine, Washington University School of Medicine in St. Louis, St. Louis, Missouri, USA. ${ }^{2}$ Center for Sleep and Circadian Neurobiology, University of Pennsylvania, Philadelphia, Pennsylvania, USA. ${ }^{3}$ National Center on Sleep Disorders Research, Division of Lung Diseases, National Heart, Lung, and Blood Institute, NIH, Bethesda, Maryland, USA. ${ }^{4}$ Douglas Mental Health University Institute, McGill University, Montreal, Quebec, Canada. ${ }^{5}$ Sleep and Chronobiology Laboratory, Department of Integrative Physiology, University of Colorado, Boulder, Colorado, USA. ${ }^{6}$ Department of Neurosciences and 'Department of Pathology, UCSD, La Jolla, California, USA. ${ }^{8}$ Department of Surgery, Washington University School of Medicine in St. Louis, St. Louis, Missouri, USA. ${ }^{9}$ Human Sleep and Inflammatory Systems Laboratory, Department of Neurology, Beth Israel Deaconess Medical Center, Boston, Massachusetts, USA. ${ }^{10}$ Division of Pulmonary, Allergy, and Critical Care Medicine, Columbia University School of Medicine, New York, New York, USA. " $C e n t e r$ for the Study of Itch, ${ }^{12}$ Department of Medicine, ${ }^{13}$ Department of Anesthesiology, ${ }^{14}$ Department of Pathology, and ${ }^{15}$ Department of Immunology, Washington University School of Medicine in St. Louis, St. Louis, Missouri, USA. ${ }^{16}$ Division of Rheumatology, Department of Medicine, Northwestern University Feinberg School of Medicine, Chicago, Illinois, USA. ${ }^{17}$ Institute for Genome Sciences, University of Maryland School of Medicine, Baltimore, Maryland, USA. ${ }^{18}$ Department of Psychiatry, UCSF, San Francisco, California, USA. ${ }^{19}$ Department of Psychology and Committee on Neurobiology, University of Chicago, Chicago, Illinois, USA. ${ }^{20}$ Department, of Anatomy, Physiology, and Cell Biology, UCD School of Veterinary Medicine, Davis, California, USA. ${ }^{21}$ Section of Infectious Diseases, Department of Internal Medicine, Yale School of Medicine, New Haven, Connecticut, USA. ${ }^{22}$ Division of Neonatology, Children's Hospital of Philadelphia, Philadelphia, Pennsylvania, USA. ${ }^{23}$ Department of Pediatrics, Perelman School of Medicine, University of Pennsylvania, Philadelphia, Pennsylvania, USA. ${ }^{24}$ Department of Biomedical Sciences, Elson S. Floyd College of Medicine, Washington State University, Spokane, Washington, USA. ${ }^{25}$ Harry S. Truman Memorial Veterans Hospital, Columbia, Missouri, USA. ${ }^{26}$ Department of Neurology, University of Missouri School of Medicine, Columbia, Missouri, USA. ${ }^{27}$ Center of Emphasis in Infectious Diseases, Department of Molecular and Translational Medicine, Paul L. Foster School of Medicine, Health Sciences Center, Texas Tech University, El Paso, Texas, USA. ${ }^{28}$ Department of Immunology and Microbiology, Scripps Research Institute, Jupiter, Florida, USA.

Conflict of interest: AAP reports financial support from Headspace Inc. YCL receives financial support from Pfizer. BSK reports support from Locus Biosciences and LEO Pharma.

Copyright: (c) 2020, American Society for Clinical Investigation.

Reference information: JCI Insight 2020;5(1):e131487.

https://doi.org/10.1172/jci.

insight.131487.
Recent discoveries demonstrate a critical role for circadian rhythms and sleep in immune system homeostasis. Both innate and adaptive immune responses - ranging from leukocyte mobilization, trafficking, and chemotaxis to cytokine release and T cell differentiation -are mediated in a time of day-dependent manner. The National Institutes of Health (NIH) recently sponsored an interdisciplinary workshop, "Sleep Insufficiency, Circadian Misalignment, and the Immune Response," to highlight new research linking sleep and circadian biology to immune function and to identify areas of high translational potential. This Review summarizes topics discussed and highlights immediate opportunities for delineating clinically relevant connections among biological rhythms, sleep, and immune regulation. 


\section{Introduction}

Circadian rhythms are daily variations in behavior and biological activity that stem from an intrinsic ability of organisms to align themselves with the environmental 24-hour light/dark cycle. These rhythms originate from an internal biological clock that drives many aspects of human physiology, including the sleepwake cycle and daily variations in blood pressure, body temperature, and cortisol (1). A growing body of epidemiological evidence demonstrates an association between altering circadian timing through shift work or frequent time zone travel and increased rates of cardiovascular disorders, metabolic syndrome, and cancer (2-4). Clinical aspects of disease such as pain perception, asthma exacerbations, and myocardial infarctions are more common at certain times of day or night $(5,6)$. The discovery of the genetic basis for the circadian clock in the 1980s and 1990s has ushered in a new era in which long-appreciated circadian rhythms in physiology and clinical medicine are being reframed in terms of gene expression, metabolism, signal transduction, and cellular physiology (7). The translation of circadian discovery into strategies to improve the prevention and management of disease promises to be transformative, but at present, fundamental research is outpacing clinical application (Figure 1A). Much will depend on research identifying the critical mechanisms and targets to which circadian rhythm-based therapeutic strategies can be applied. An emerging example of exciting circadian discovery with potential clinical relevance is the intersection between circadian function and immune regulation (Figure 1B).

The NIH recently sponsored a workshop entitled "Sleep Insufficiency, Circadian Misalignment, and the Immune Response" (May 16-17, 2019, Rockville, Maryland, USA). Its aim was to highlight basic and clinical advances linking sleep and circadian biology to immune dysfunction, thereby stimulating the application of circadian biology to translational medicine. The Workshop was cosponsored by four NIH institutes - the National Heart, Lung, and Blood Institute (NHLBI), National Institute on Aging (NIA), National Institute of Allergy and Infectious Diseases (NIAID), and National Institute on Alcohol Abuse and Alcoholism (NIAAA) - reflecting a broad interest and recognizing that circadian and sleep-based research cuts across traditional academic disciplines. Here, we report the perspective of the Workshop participants on circadian biology, sleep, and immunity, and review emerging literature that links circadian rhythms and sleep to specific immune functions. Finally, we highlight future research opportunities as well as challenges that need to be overcome in order to implement chronotherapy in clinical settings.

\section{Introduction to the molecular circadian clock}

Circadian rhythms evolved as organisms were selected for their preparedness for the environmental shifts brought about by the solar day (8). The molecular model for circadian rhythm generation arose from genetic screens designed to find mutations that disturb the sleep-wake cycle in model organisms (7). In both flies and mammals, the key constituents of the molecular circadian clock are either transcription factors or transcription factor regulators (i.e., "clock genes"; Figure 2). At the core of the clock is a heterodimer composed of the proteins BMAL1 and CLOCK (9). This complex stimulates transcription by altering the chromatin landscape around genes containing E-box motifs in their promoters (10). Among the downstream effectors of BMAL1/CLOCK are fellow clock gene products Per1-3, Cry1/2, NR1D1/2 (also known as REV-ERB $\alpha / \beta$, and ROR $\alpha-\gamma$, whose products either negatively or positively regulate the core complex (9). Thus, the expression of clock genes oscillates with roughly a 24-hour periodicity, thereby biochemically representing the solar day. Other downstream effectors of the molecular clock include master transcription factors, such as the PAR domain basic leucine zipper transcription factor family (DBP, TEF, and HLF) (11), nuclear factor IL-3-regulated (NFIL3, also known as E4BP4) (12), and the PPAR family $(11,13)$. Through these clock-controlled genes (CCGs) as well as others, the molecular clock imparts a circadian pattern on gene expression, and by extension protein and metabolite abundance. Molecular clocks operate in almost all nucleated cells throughout the body, thereby allowing cells to factor timeof-day information into the control of metabolism and other key pathways (14).

The molecular clock contains some special features that are important for its role in regulating the physiology of higher organisms. First, clock proteins and clock genes are subject to numerous forms of biochemical regulation, including acetylation, methylation, phosphorylation, ubiquitination, and sumoylation (15). Combined with signal transduction through accessory pathways, these modifications impact clock protein levels and activity, thereby allowing ambient biochemical cues, such as temperature, nutrient status, hormone levels, and innervation, to synchronize internal cellular time with the external world (16). This phenomenon is known as entrainment and serves an additional crucial role in creating temporal coherence within organs by synchronizing parenchymal cells with one another. To harmonize the various biochemical entrainment 


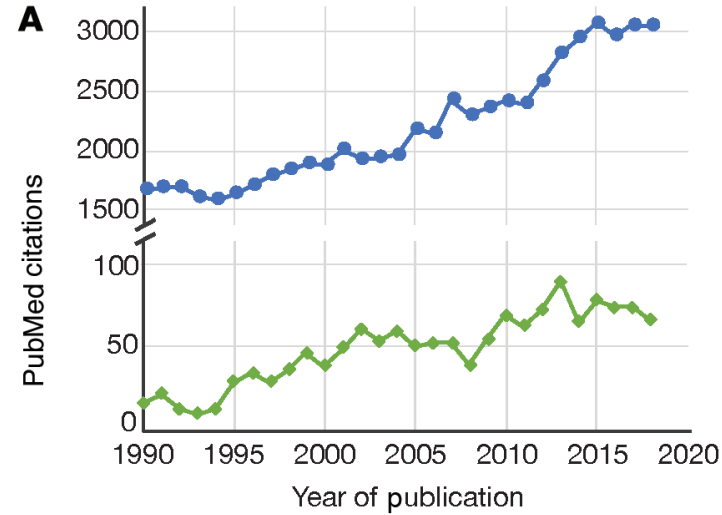

Circadian

Chronotherapy
B

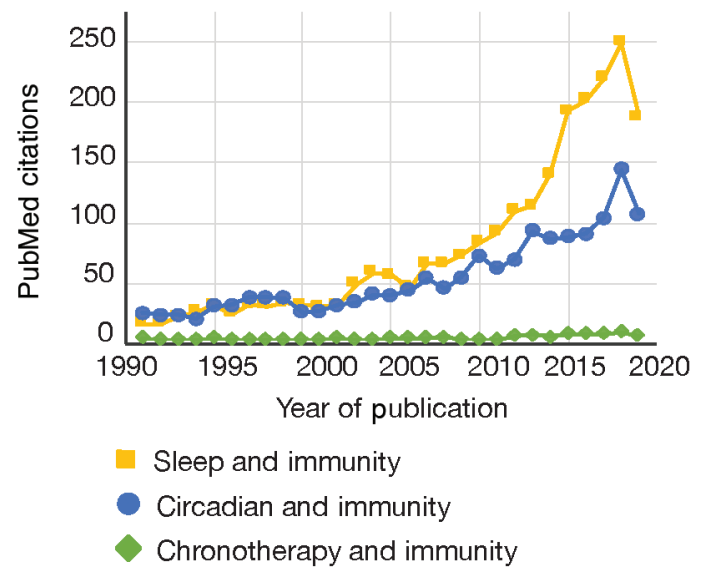

Figure 1. Citations in chronotherapy and circadian research are on the rise. (A) Number of 1990-2018 publications found with PubMed searches for "circadian" and "chronotherapy." (B) Number of 1990-2018 publications found with PubMed searches for "sleep and immunity," "circadian and immunity," and "chronotherapy and immunity." Chronotherapy is classically defined as the use of circadian information to maximize the therapeutic index of a medical intervention or to limit the amount of drug needed to achieve a clinical end point by giving it at the optimal time of day. An emerging use of the term is for the direct targeting of clock gene function to achieve a clinical end point, such as tumor killing (148). Illustrated by Rachel Davidowitz.

cues, mammals require a "master circadian pacemaker" located in the suprachiasmatic nucleus (SCN) of the hypothalamus. The SCN receives light information from the retina and then projects to other CNS areas that regulate arousal, autonomic tone, temperature, and hormone secretion (16). Of all the entrainment cues, light has the largest impact on rest-activity rhythms, and the clocks within SCN neurons are selectively sensitive to light for entrainment. However, because SCN clocks synchronize to changing light cycles faster than peripheral clocks, and because mistimed feeding or other biochemical cues can compete with SCN pacing in the periphery (17), it is possible to induce a state of internal desynchronization. This desynchronization is thought to explain the sequelae of jet lag and night-shift work (18-20). A second key feature of the molecular clock is that its regulatory influence extends from the microscopic to the macroscopic biological scale. For example, mutations that alter the periodicity of $\mathrm{Per}$ and $\mathrm{Cry}$ expression provoke analogous changes to the periodicity of sleep timing and quality (21-23). A final feature is programmability. While the anatomy of the molecular clock is largely uniform across tissues, the circadian transcriptomes it generates (i.e., the set of CCGs exhibiting rhythmic expression) are highly tissue specific and overlap by only about $10 \%$ between organs (24). The remarkable organ specificity of circadian transcriptomes is thought to be a product of cell type-specific transcription factors and tissue-selective patterns of chromatin accessibility $(25,26)$. Collectively, the cardinal molecular clock features of entrainment, programmability, and the ability to impact function at all biological scales enable tissues to harness time-of-day information in the service of their unique physiologic contributions. They also makes it possible for the clock to organize diverse networks of cells around a common goal, for example, a coordinated immune response.

\section{Clocks and immunity}

The current focus on circadian control of immune function was presaged by the observations - first published more than 50 years ago - that circulating lymphocyte counts oscillate on a daily basis in healthy humans (27) and that the susceptibility to endotoxin depends on the time of day it is administered in rodents (28). More recent work highlights epidemiological associations between night-shift work (i.e., activity and sleep patterns in opposition to the normal day-night pattern) and elevated levels of systemic inflammatory markers $(29,30)$. Significantly, all lymphoid organs and immune cell types tested to date harbor functional clocks $(31,32)$. Studies using genetic experimental approaches in human cells and mice suggest a connection between clocks and at least three fundamental immunologic activities.

The best-documented connection between clocks and immune function is the regulation of proinflammatory cytokine secretion. Cytokines are well-known mediators of host responses to infection, trauma, and immune responses. In endotoxin-stimulated macrophages, the magnitude of TNF- $\alpha$ secretion was found to vary in a circadian fashion based on the time of endotoxin challenge (31). The current paradigm for 


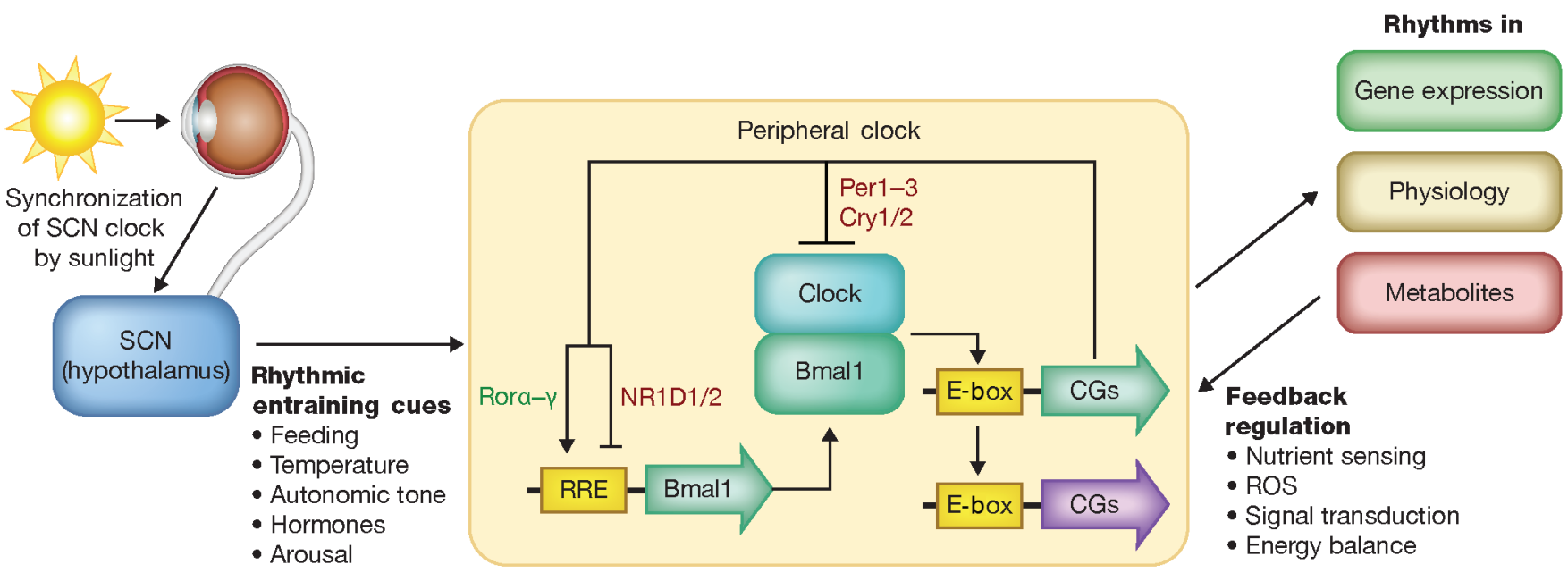

Figure 2. Circadian regulation in mammals. Schematic depicting the currently accepted hierarchal model for circadian rhythm generation. Light information is conveyed by the optic nerve to the SCN, a region of the ventral hypothalamus. There, light entrains clocks within SCN neurons, and this is ultimately converted by the CNS into pulsatile chemical and neurological cues, which entrain cell-autonomous circadian clocks residing in peripheral cells. These peripheral clocks impart circadian patterns on gene expression and overall cellular physiology. For simplicity, only the core molecular clock circuitry is depicted, with positive regulatory proteins labeled green and negative regulators labeled red. However, there are many accessory proteins and metabolic pathways that can adjust the periodicity and phase of the clock but are not central to rhythm generation (for example, casein kinase 1 $\delta / \varepsilon$ [ref. 23], AMPK [ref. 149], mTOR [ref. 150], p53 [ref. 151], and SIRT1 [ref. 152]). There are additional molecular clock constituents (not depicted) that in the basal state appear to have more prominent roles in CNS clocks than clocks in peripheral cells. For example, NPAS2 (a functional homolog of CLOCK) and DEC1/2 provide additional negative feedback to BMAL1/CLOCK $(16,153)$. Yellow boxes represent E-boxes or ROR-responsive elements (RREs), which are the promoter motifs recognized by BMAL1/CLOCK or REV-ERB/ROR proteins, respectively. Clock genes and clock-controlled genes (CGs) are represented by green and purple arrows, respectively. Illustrated by Rachel Davidowitz.

explaining how the clock imparts rhythms to cytokine production involves the ability of clock proteins to directly transactivate or repress gene expression of key cytokines and chemokines, including CCL2, TNF, IL-6, and CXCL5 (reviewed in ref. 33). This paradigm also extends to CCGs. For example, in LPS-stimulated macrophages, the clock-controlled transcription factors NFIL3 and DBP impart a circadian rhythm to IL-12(p40) secretion by alternating their binding to the Il12 promoter throughout the day (34). Thus, disruption of molecular clock function through light-cycle desynchronization or deletion of Bmall or Rever$b a$ locks macrophages into a "time of day" wherein inflammatory cytokine secretion is maximized (35, 36). This hypersecretory phenotype is not limited to immune cells but extends to epithelial cells, which are responsible for initial leukocyte recruitment after infectious and sterile insults (36-38). Interestingly, inflammation appears to feed back on the integrity of the molecular clock. For example, Clock, REV-ERB $\alpha$, and BMAL1 protein levels decline rapidly in cells after endotoxin treatment, which is predicted to disrupt clock function (39-41). Conversely, herpes virus infection stimulates Bmall promoter activity, leading to a corresponding depression in Cry 1 and a disruption in the feedback relationships that underlie the clock (42). Indeed, disruption of clock gene expression appears to be a common outcome of acute infection and leads to novel rhythms of gene expression characterized by prominent cycling of proinflammatory genes $(37,43)$. Some suggest that the circadian clock is itself a kind of innate immune sensor that when disabled by infection automatically shifts cells to a proinflammatory state. However, during chronic inflammation or stressors such as shift work, disrupted clocks have been shown to increase the morbidity in response to infection or sterile insult through excessive inflammation (44-49).

Trafficking of myeloid and lymphocyte subsets in healthy mice and humans is another circadian-regulated immune activity (50-52). Leukocyte trafficking represents the summation of several discrete, individually rhythmic processes (Figure 3 ), including cell egress from bone marrow $(53,54)$, leukocyte homing from the bloodstream to the interstitial space of organs $(51,55)$, and removal of leukocytes from organs through phagocytosis or via transit to regional lymph nodes $(56,57)$. Regardless of cell type, mechanisms of rhythmic trafficking involve coordinated clock function within the leukocyte and within endothelial cells lining the vessels of peripheral organs. Together, these clocks align the expression of receptor-ligand pairs critical for chemoattraction and adhesion to the endothelial surface, thereby facilitating leukocyte recruit- 
ment $(51,55)$. Additional levels of control, including adrenergic tone and leukocyte ROS, appear to play a role in setting the phase of leukocyte trafficking rhythms $(51,52,58)$. Interestingly, the critical ligand-receptor interactions needed for leukocyte homing appear to vary depending on the cell type and the destination organ (55). Altogether, the complexity of leukocyte trafficking makes clear the importance of the clock in optimizing this critical immune activity.

A third connection between clocks, clock genes, and immune processes is among the least studied: differentiation and maturation of clinically important leukocyte subsets. For example, the canonical clock gene Rora is required for the differentiation of type 2 innate lymphoid cells (ILC2s), which play a role in type II inflammation, allergy, and defense against parasites (59). A splice variant of the clock gene Rorg (Rorgt) is critical for the development of type 3 innate lymphoid cells (ILC3s), lymphoid tissue inducer (LTi) cells, and the $\mathrm{CD}^{+} \mathrm{T}$ helper cell subset Th17 cells (60-62). Rora is also important for the full development of Th17 cells (63). ILC3s and Th17 cells are characterized by secretion of IL-17, and both are implicated in autoimmune diseases. Disruption of molecular clock function leads to aberrant Th17 cell development and renders mice more susceptible to pathology in models of autoimmunity, including experimental autoimmune encephalomyelitis (EAE), a mouse multiple sclerosis model, and colitis $(64,65)$. Interestingly, Rorgt mRNA expression is blocked by melatonin, a hormone with pleiotropic immunomodulatory activity that is secreted in a circadian fashion by the pineal gland $(66,67)$. Finally, circadian clocks were recently found to regulate neutrophil maturation (68), which is remarkable given the short life span (only 1-2 days) of these crucial effector cells. As neutrophils age, they undergo cytoskeletal and cell-adhesive changes that enhance their migration into the interstitial spaces of organs according to a circadian rhythm $(53,68)$. These changes are mediated by Bmall, and loss of this clock gene blocks their efficient homing to organs (68). The result is that initial innate responses against invading organisms are weakened in Bmall-deficient mice, thereby increasing disease severity (68). Clock control of neutrophil maturation has an additional homeostatic function, as trafficking of aged neutrophils into the bone marrow suppresses the hematopoietic niches that control the egress of leukocytes in the first place (53). In doing so, rhythmic neutrophil homing completes a feedback loop that may help to make circadian trafficking patterns for all hematopoietic types self-sustaining.

Altogether, it is increasingly clear that circadian gating is part of the core programing of the immune system, and thus, alteration of this regimen is likely to have widespread ramifications for disease pathogenesis. Current evidence draws a direct mechanistic line between the biochemistry of a clock protein, such as BMAL1, and a discrete immune parameter, like the production of the neutrophil chemoattractant CXCL5 during lung injury (36). Alternatively, the clock may impact immunity by regulating sleep.

\section{Sleep and immunity}

Sleep is a basic behavior common to almost all organisms and is defined by a period of relative unresponsiveness to outside stimuli. In humans, sleep can be subdivided into an orderly progression of neurological stages based on EEG patterns that repeat roughly every 90 minutes (69). There is no consensus on what defines ideal sleep, but total sleep deprivation in rodents is fatal (70). It is still not fully understood why denying rodents sleep is deadly; however, studies have shown that chronically sleep-deprived animals exhibit splenic atrophy and polymicrobial bacteremia, suggesting that immune dysfunction may be part of the dying process $(70,71)$. In a similar vein, short habitual sleep ( $<6$ hours per night) is statistically associated in humans with reduced life span, increased vulnerability to viral infection, and reduced antibody titers after vaccination (72-74). Short-term sleep deprivation prior to vaccination appears to negatively impact antibody titers after influenza vaccination and, at least in rodents, reduces influenza vaccine efficacy (75-77). Just as sleep impacts immune functions, it appears that certain peripheral immune populations may affect sleep. For example, chemical or genetic ablation of macrophages affects the architecture of rebound sleep in sleep-deprived mice $(78,79)$. Altogether, sleep is connected to an organism's resilience against infection, and considerable work has been done in humans and animal models to examine how sleep deprivation impacts specific immune parameters (80).

The relationship between circadian rhythms and sleep is complex and likely bidirectional. In humans, the paradigm for sleep timing is a "two-process" model (processes C and S) in which CNS circadian clocks play a major role. Clocks generate daily oscillations in neurological arousal (process $\mathrm{C}$ ), which promotes wakefulness and opposes drowsiness that accumulates through sleep debt (process S) (81). Work in Bmal1-deficient model organisms suggests that clocks may have an additional role in mediating normal sleep architecture (82- 

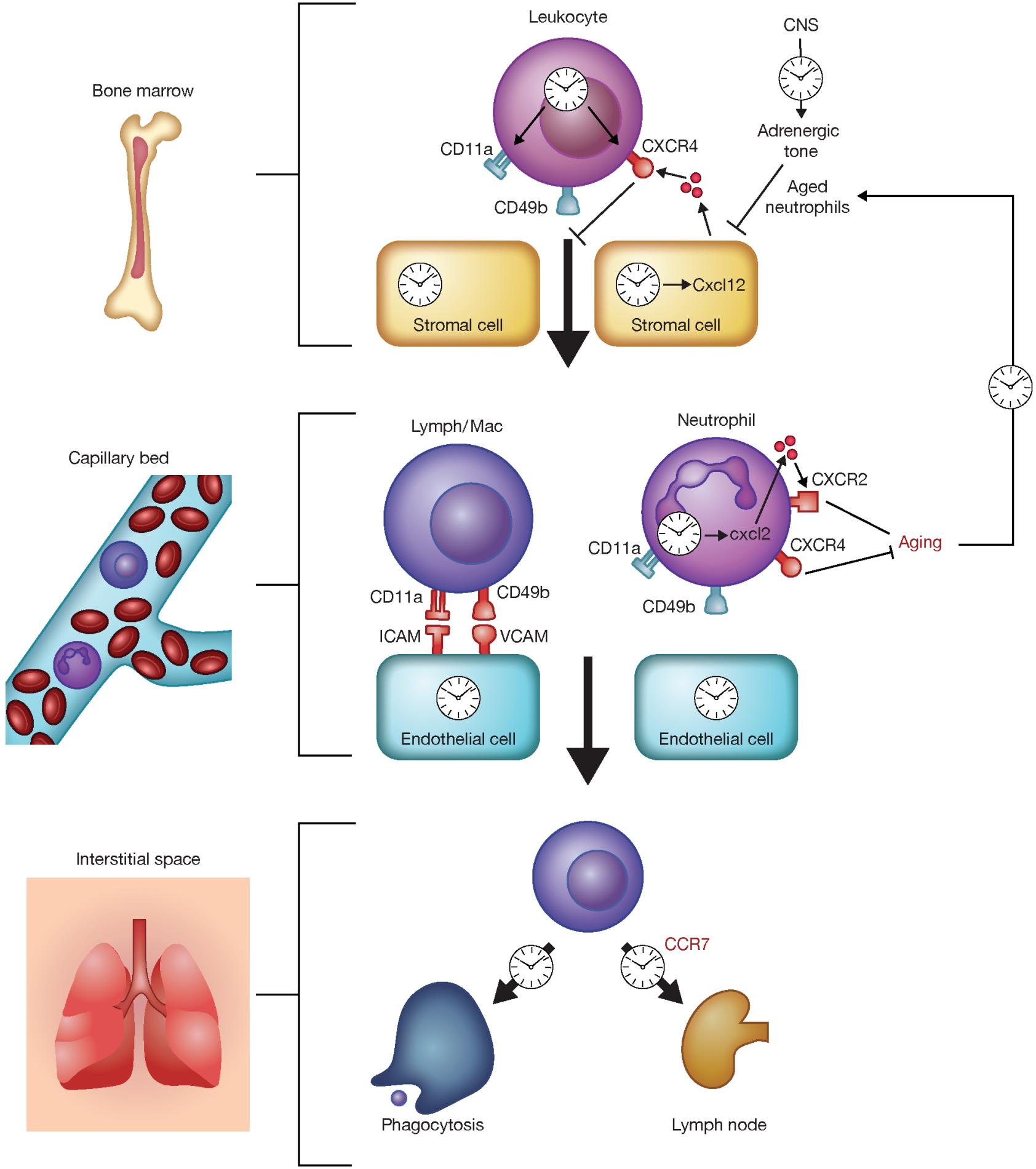

Phagocytosis

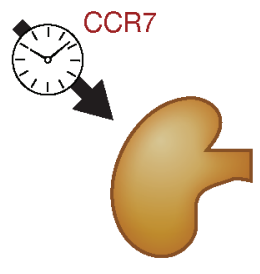

Lymph node

Figure 3. Multilayered circadian control of leukocyte trafficking. Schematic depiction of the circadian regulation of specific leukocyte trafficking steps $(55-57,68)$. These include the egress of leukocytes from the bone marrow; adhesion of circulating leukocytes to endothelial cells in the capillary beds of end organs; and removal of leukocytes from the parenchyma of organs by phagocytosis or by migration to area lymph nodes. Proteins generally important for rhythmic leukocyte trafficking at specific steps in the process are depicted in red. For a comprehensive treatment of cell-specific determinants of leukocyte trafficking rhythms, see Pick et al. (154). Lymph/Mac, lymphocyte/macrophage. Illustrated by Rachel Davidowitz. 
84). While clocks gate sleep, it is also true that short-term sleep disruption can affect circadian regulation in peripheral tissues such as blood and lung, by either damping the rhythms in CCG expression or altering their phase $(85,86)$. Thus, while circadian rhythms and sleep are biologically different phenomena and can be analyzed in isolation using "forced synchronization" protocols in humans (87), it is difficult to perturb one without affecting the other under naturalistic conditions. For physicians, the conflation of circadian rhythms and sleep during disease dates to Greek antiquity. The writings of Arataeus of Cappadocia on asthma posed the question of whether asthma exacerbations are more frequent at night because of the time of day or because patients are typically asleep at night (88). Some pathogens even corrupt the circadian-mediated sleep/wake cycle to promote disease. For example, Trypanosoma brucei (which causes African sleeping sickness) alters sleep patterns in afflicted subjects by shortening the periodicity of the molecular circadian clock (89).

Given the interconnectedness of sleep and circadian rhythms, it is understandable that the immune activities affected by insufficient sleep overlap with those altered by circadian disruption. Experimentally induced sleep deprivation and short habitual sleep increase proinflammatory cytokine secretion, particularly in males $(90,91)$. Numbers of circulating neutrophils, NK cells, monocytes, and B cells are increased by prolonged wakefulness and decreased by recovery sleep, suggesting a role for sleep in regulating leukocyte trafficking $(80,92)$. Recent work in mice found that sleep fragmentation increases the production of inflammatory monocytes by the bone marrow and leads to atherosclerosis, an inflammatory pathology also induced by Bmal1 deficiency $(93,94)$. Finally, inadequate sleep seems to impair immune effector cell functions such as NK cell activity, at least in some experimental scenarios $(80,95)$.

Circadian rhythms and sleep are increasingly seen as important for immune system homeostasis. Disruption of either process can engender a state of inflammation and functional immunocompromise, rendering organisms more vulnerable to disease. Historically, circadian biology, sleep, and immunology have occupied separate branches of investigation. Consequently, there has been little interdisciplinary research on these topics. The key point is that circadian rhythm and sleep disruption happen concurrently every day through the widespread experiences of shift work, nighttime light exposure, and social jet lag, which is caused by discontinuity between natural sleep-wake schedules and the variations in the social demands of weekdays versus weekends. Thus, the interface between circadian rhythms, sleep, and immune function potentially has a wide range of implications for public health and medicine.

\section{Workshop-identified opportunities}

The goal of the NIH Workshop was to highlight recent basic and clinical research advances in sleep and circadian biology linking immune dysfunction to pathobiology, and to foster interdisciplinary conversations that could point toward future research objectives. Following 2 days of presentations and discussions covering a wide range of disease-based research, the participants developed recommendations on the most timely and compelling questions in this area. The participants also discussed various challenges for circadian-immune and sleep-immune discovery research to be translated and implemented more effectively.

Adaptive immune response. The highest-priority knowledge gap identified by Workshop participants was the need to better understand the role of circadian rhythms and sleep in adaptive immune responses. While there has been an intense focus on how clocks regulate innate immunity, research into the mechanisms connecting circadian and sleep disruption to adaptive immune functions remains sparse. This is partly due to early skepticism that something as short as a 24-hour clock could be relevant to antigen-specific B and T cell responses, which form over days to weeks. Results from prior experiments in mice with T cell-specific Bmal1 deletion were interpreted to mean that circadian clocks have little influence over adaptive immune activities (96). However, some of these earlier experiments did not factor time of day into the experimental design, and subsequent studies that controlled for this variable did report circadian effects in outcomes such as autoimmune pathology $(56,65)$. Moreover, $\mathrm{T}$ cell proliferation in response to antigen displays a circadian rhythm in mice that is clock dependent (97). In other research, time of day and sleep quantity were both shown to impact markers of vaccine efficacy in humans and rodents (44, 74, 75, 97-99). Clocks may also affect adaptive immunity via control of immune checkpoints, as disruption of circadian rhythms in rodents appears to exacerbate bacterial peritonitis in part by increasing programmed cell death ligand 1 (PD-L1) expression on macrophages, which represses T cell activities (100). With this background, Workshop participants identified a critical need to elucidate how circadian clocks mediate various aspects of specific immunity. The potential clinical ramifications of such research are very broad, including, but not limited to, the treatment of autoimmunity (101), long-term preservation of organ function in transplanted organs 
such as the lung $(102,103)$, cancer immunotherapy (104-106), establishment of allergic tolerance (107), "immune paralysis" that accompanies severe sepsis $(100,108)$, and even age-related changes in immune function $(109,110)$. A specific opportunity with high potential impact for public health is to achieve a better understanding of how the timing of immunization might influence the molecular and cellular processes underlying the establishment of protective immunity. Such research could find application in improving existing immunization programs in the elderly and, importantly, in targeting diseases deemed to be difficult targets for vaccination, such as malaria (111-114).

Neuroinflammatory mechanisms. Examination of neuroinflammatory mechanisms as a link by which circadian rhythms and sleep affect immunity was identified as another topic of interest. It is increasingly appreciated that the nervous system and immune system share common mediators. For example, Th2 cytokines, such as IL-4 and IL-13, figure prominently in the pathogenesis of allergy and asthma but also serve as neurotransmitters within the peripheral nervous system, where they mediate itch $(115,116)$. Acetylcholine is a classic neurotransmitter of the CNS and the autonomic nervous system but is also secreted by a subpopulation of peripheral T cells $(117,118)$. Importantly, chronic itch has a strong nocturnal predominance, and autonomic tone has a prominent circadian rhythm $(51,119)$. Moreover, clock gene deletion appears to exacerbate neurodegenerative disease in experimental models in part by inducing inflammatory responses in astrocytes $(120,121)$. Conversely, neurodegenerative diseases are accompanied by coincident disturbances in sleep and circadian activity patterns, implying a bidirectional relationship (122). Exploring potential circadian and sleep influences on neuroimmune pathways inside and outside the CNS could translate into new therapeutic approaches for a wide variety of human diseases. One opportunity cited by Workshop participants was to better understand how circadian rhythms in autonomic tone contribute to neuroimmune reflexes, such as those that influence sepsis outcomes $(123,124)$. Results of such research could find application in the treatment of critical illnesses such as sepsis and pneumonia potentially by targeting the molecular clock and its downstream effectors, or by optimally timing interventions that target the inflammatory reflex. Another potential application is targeting the clock to reduce neuroinflammation that contributes to Alzheimer's disease and other neurodegenerative disorders (120-122).

Microbiome and immunity. A third high-impact opportunity identified by Workshop participants is to better understand interrelationships among circadian rhythms, the microbiome, and immunity. The microbiome - made up of trillions of microorganisms found throughout the body, with the largest numbers in the small and large intestines - is unique to each individual and plays a significant role in health and disease. Current research suggests a complex bidirectional relationship between circadian clocks and the microbiome, which produces metabolites that modulate innate and adaptive immune responses (125-127). Rodents exhibit daily rhythms in microbiome mass that are driven by circadian patterns in feeding behavior $(128,129)$. In turn, normal microbiota are necessary for normal circadian gene expression in the liver (130). Therefore, circadian-mediated release of microbiota-derived metabolites may directly regulate rhythmic immune-related phenotypes. Rhythmic release of metabolites may also synchronize the body to the external environment, possibly extending to the entrainment of immune cells. One specific opportunity is to elucidate the relationship among the microbiota, the circadian rhythm, and immune responses, with obvious applications for gastrointestinal disease. Since metabolic and immune responses are often linked, understanding the interplay among the microbiome, the immune system, and circadian rhythms would also be applicable in the treatment of metabolic syndrome, an important risk factor for both cardiovascular and endocrine diseases (131).

Additionally, the participants encouraged applying circadian and sleep biology to topics for which such influences are underexplored. Leading these were the fetal origins of adult-onset disease (94), sex-specific differences in various disease models, substance abuse $(132,133)$, crosstalk between innate and adaptive immune cells, and the ability of immune cells to induce physiological changes in nonimmune cells. A recent study suggested that circadian gene expression in mouse lungs could be reprogrammed by depleting mice of neutrophils, even though these cells are relatively uncommon in the lung parenchyma (134). It is likely that there are other examples of immune end-organ crosstalk that may have physiologic or medical significance.

\section{Challenges}

Circadian and sleep biology hold considerable promise for the development of immunomodulatory therapies to combat disease. However, there was agreement among the Workshop participants that scientific, technical, and cultural challenges must be overcome if chronotherapy is to be applicable in a general clinical setting. 
Scientific challenges. We are living through a great era of molecular genetics, in which the ability to modulate the expression of single genes is transforming the way we understand disease. The discovery of the molecular circadian clock might never have occurred without genetic screens in Drosophila (7), and sleep research also benefits from the application of genetics (135). Unquestionably, knockout mice and other genetic reagents represent important stepping-stones for examining circadian-sleep-immune connections, but exclusive reliance on genetic approaches in model organisms is not without caveats. While the use of small molecule modulators should complement genetic approaches, caution should be exercised with them too. This is due to a combination of factors, including complexity of the immune system, multiple cell types and peripheral tissues involved, and genetic redundancy in the circadian clock. Thus, exclusive reliance on either genetic or chemical techniques to infer mechanism can be problematic. Fortunately, there are diverse ways, including genetic, environmental, and chemical approaches, to interfere with circadian and sleep research activities (136-139). Overall, the Workshop favored a philosophy of routine orthogonal validation (i.e., validating conclusions using at least two technically disparate experimental approaches) to better understand how circadian clocks, sleep, and immunity affect each other.

Technical challenges. One important technical need for facilitating trials incorporating circadian biology is a quick and robust way of determining the circadian phase of an individual (biological time of day versus external time). The reason for this is that in developed regions, nearly every individual is at risk of desynchrony due to modern-day infrastructure (i.e., artificial light, social media, irregular sleep-wake schedules, time zone changes, etc.). This is evident from prospective observations of nurses whose circadian rhythms in body temperature were shown to be out of phase as much as 3 days after a night shift (140). On the gene expression level, analyses of human circadian transcriptomes from biopsy samples suggest that about $20 \%$ of the populace is significantly out of phase relative to the majority, perhaps reflecting the prevalence of shift work in developed countries (141). What this means is that any chronotherapy trial wherein an intervention is pegged to a set time of day must contend with the fact that the internal biological time of patients involved can be quite different. Future attempts to translate circadian biology to the clinic will need to focus on ways of defining the circadian phase of each participant in advance so that the therapy is applied at the optimal time for each individual. To this end, it would be valuable to include a run-in period where sleep hygiene and uniform circadian entrainment are pursued as a goal before a chronotherapy intervention is attempted. However, Workshop participants noted that requiring a run-in period for chronotherapy trials could discourage future research because of the increased cost and study complexity this practice would incur. Fortunately, recent work on circadian biomarkers suggests that spot analysis of gene expression in blood samples can determine patients' internal circadian time, which could potentially offset the need for a run-in period $(142,143)$. Similarly, robust and quantifiable biomarkers of sleep debt would greatly facilitate translational circadian rhythm research, as sleep disturbance may mask the immunologic benefits of a chronotherapy intervention.

Another technical challenge is that circadian amplitudes vary dramatically between individuals (49), but the significance of this variation is not known. At the gene expression level, all circadian transcriptomes compiled to date represent average gene expression across a tissue sample or collection of cells. When the amplitude of clock gene expression is found to be relatively low (as occurs after shift work; ref. 144), it could mean that clock gene expression has been identically damped in each individual cell, or that the cellular clocks in the sample have fallen out of phase with one another so that the average of the signals is low. To decipher the meaning of circadian amplitude changes, profiling of circadian rhythms at the single-cell level will likely be required. To generate high-quality circadian transcriptomes from mammalian tissue, RNA-sequencing (RNA-Seq) reads on the order of 20-40 million per sample are needed (145), but current single-cell sequencing technology delivers on the order of thousands of reads per cell. Technical improvements that extend the reading depth of single-cell RNA-Seq would foster its application to circadian measurements in humans and could greatly speed translational research.

Cultural challenges. In addition to posing scientific and technical challenges, chronobiology runs against the grain in much of the developed world, which is designed to liberate people from natural biological rhythms. Many may enjoy the convenience and stimulation offered by the so-called 24-7 society. Nighttime illumination renders streets safer, and in a culture where people consider time a precious commodity, sleep can be construed as wasteful. Specific to the patient experience, the process of care in hospitals is designed around the rapid provision of diagnostic services and medical intervention, and thus circadian rhythms and sleep quality are inevitably disregarded. Nevertheless, biological sleep and consolidated circa- 
dian rhythms are essential for health, and a greater effort to communicate scientific findings to the general public and the medical community is urgently needed. Ultimately, societies may or may not be prepared to make changes in, for example, how hospitals are run or the adoption of time-release drug preparations that are more expensive in the short term but confer superior medical and economic utility in the long term (146). Regardless of these meta-issues, small innovations can yield significant benefits. For example, simply recording the times immunologic assays are conducted may improve the reproducibility of preclinical research in some circumstances, even when circadian rhythms are not the intended focus of study $(34,147)$. Another recommendation raised by Workshop participants was to modify preclinical toxicology studies such that new investigational drugs are dosed at different times of day. This might help reduce side effects in phase I clinical trials, leading to a more streamlined approval process.

\section{Conclusion}

Emerging intersectional research on sleep, circadian rhythms, and immunity presents exciting opportunities for using scientific understanding to augment the immune resilience of shift workers and other at-risk, but otherwise healthy, personnel, while at the same time mitigating the burden of major inflammatory diseases. A better understanding of how the immune system utilizes time as a biological variable could engender the development of new immunomodulatory therapies and the timing of administration of therapies, all of which may help improve the efficacy of existing drugs. A deeper understanding may also aid in the development of regimens to maximize vaccine efficacy, combat infections, and optimize time of organ transplant to minimize rejection. While connections to adaptive immunity and neuroinflammatory reflexes represent some highly opportune areas for study in the present, there are many areas of disease physiology for which the insights of circadian and sleep biology have yet to be considered. We may discover that circadian rhythms are an essential clinical element that must be factored into health and prevention efforts, as well as the diagnosis and therapeutics of disease.

\section{Acknowledgments}

The authors wish to thank the NHLBI, NIA, NIAID, and NIAAA for organizing and supporting the Workshop.

Address correspondence to: Jeffrey Haspel, Division of Pulmonary and Critical Care, Washington University School of Medicine in St. Louis, 660 South Euclid Avenue, St. Louis, Missouri, USA. Phone: 314.362.4112; Email: jhaspel@wustl.edu.

1. Refinetti R. Integration of biological clocks and rhythms. Compr Physiol. 2012;2(2):1213-1239.

2. Kecklund G, Axelsson J. Health consequences of shift work and insufficient sleep. BMJ. 2016;355:i5210.

3. Kwon P, et al. Night shift work and lung cancer risk among female textile workers in Shanghai, China. J Occup Environ Hyg. 2015;12(5):334-341.

4. Schernhammer ES, Feskanich D, Liang G, Han J. Rotating night-shift work and lung cancer risk among female nurses in the United States. Am J Epidemiol. 2013;178(9):1434-1441.

5. Smolensky MH, et al. Diurnal and twenty-four hour patterning of human diseases: cardiac, vascular, and respiratory diseases, conditions, and syndromes. Sleep Med Rev. 2015;21:3-11.

6. Glynn CJ, Lloyd JW. The diurnal variation in perception of pain. Proc R Soc Med. 1976;69(5):369-372.

7. Huang RC. The discoveries of molecular mechanisms for the circadian rhythm: the 2017 Nobel Prize in Physiology or Medicine. Biomed J. 2018;41(1):5-8.

8. Kuhlman SJ, Mackey SR, Duffy JF. Biological Rhythms Workshop I: introduction to chronobiology. Cold Spring Harb Symp Quant Biol. 2007;72:1-6.

9. Mackey SR. Biological Rhythms Workshop IA: molecular basis of rhythms generation. Cold Spring Harb Symp Quant Biol. 2007;72:7-19.

10. Trott AJ, Menet JS. Regulation of circadian clock transcriptional output by CLOCK:BMAL1. PLoS Genet. 2018;14(1):e1007156

11. Gachon F, Olela FF, Schaad O, Descombes P, Schibler U. The circadian PAR-domain basic leucine zipper transcription factors DBP, TEF, and HLF modulate basal and inducible xenobiotic detoxification. Cell Metab. 2006;4(1):25-36.

12. Cowell IG. E4BP4/NFIL3, a PAR-related bZIP factor with many roles. Bioessays. 2002;24(11):1023-1029.

13. Chen L, Yang G. PPARs integrate the mammalian clock and energy metabolism. PPAR Res. 2014;2014:653017.

14. Green CB, Takahashi JS, Bass J. The meter of metabolism. Cell. 2008;134(5):728-742.

15. Lee Y, Kim K. Posttranslational and epigenetic regulation of the CLOCK/BMAL1 complex in the mammalian. Anim Cells Syst (Seoul). 2012;16(1):1-10

16. Schibler U, et al. Clock-talk: interactions between central and peripheral circadian oscillators in mammals. Cold Spring Harb Symp Quant Biol. 2015;80:223-232. 
17. Stokkan KA, Yamazaki S, Tei H, Sakaki Y, Menaker M. Entrainment of the circadian clock in the liver by feeding. Science. 2001;291(5503):490-493.

18. Reinberg A, Ashkenazi I. Internal desynchronization of circadian rhythms and tolerance to shift work. Chronobiol Int. 2008;25(4):625-643

19. Vosko AM, Colwell CS, Avidan AY. Jet lag syndrome: circadian organization, pathophysiology, and management strategies. Nat Sci Sleep. 2010;2:187-198.

20. Reinberg A, et al. Circadian time organization of professional firemen: desynchronization-tau differing from 24.0 hours-documented by longitudinal self-assessment of 16 variables. Chronobiol Int. 2013;30(8):1050-1065.

21. Patke A, et al. Mutation of the human circadian clock gene cry1 in familial delayed sleep phase disorder. Cell. 2017;169(2):203215.e13.

22. Toh KL, et al. An hPer2 phosphorylation site mutation in familial advanced sleep phase syndrome. Science. 2001;291(5506):1040-1043.

23. Xu Y, et al. Functional consequences of a CKIdelta mutation causing familial advanced sleep phase syndrome. Nature. 2005;434(7033):640-644.

24. Zhang Y, et al. Discrete functions of nuclear receptor Rev-erba couple metabolism to the clock. Science. 2015;348(6242):14881492.

25. Caratti G, et al. REVERBa couples the circadian clock to hepatic glucocorticoid action. J Clin Invest. 2018;128(10):4454-4471

26. Beytebiere JR, et al. Tissue-specific BMAL1 cistromes reveal that rhythmic transcription is associated with rhythmic enhancer-enhancer interactions. Genes Dev. 2019;33(5-6):294-309.

27. Elmadjian F, Pincus G. A study of the diurnal variations in circulating lymphocytes in normal and psychotic subjects. J Clin Endocrinol Metab. 1946;6:287-294.

28. Halberg F, Johnson EA, Brown BW, Bittner JJ. Susceptibility rhythm to E. coli endotoxin and bioassay. Proc Soc Exp Biol Med. 1960;103:142-144.

29. Amano H, Fukuda Y, Yokoo T, Yamaoka K. Interleukin-6 level among shift and night workers in japan: cross-sectional analysis of the J-HOPE Study. J Atheroscler Thromb. 2018;25(12):1206-1214.

30. Puttonen S, Viitasalo K, Härmä M. Effect of shiftwork on systemic markers of inflammation. Chronobiol Int. 2011;28(6):528-535.

31. Keller M, et al. A circadian clock in macrophages controls inflammatory immune responses. Proc Natl Acad Sci U S A. 2009;106(50):21407-21412.

32. Silver AC, Arjona A, Hughes ME, Nitabach MN, Fikrig E. Circadian expression of clock genes in mouse macrophages, dendritic cells, and B cells. Brain Behav Immun. 2012;26(3):407-413.

33. Scheiermann C, Gibbs J, Ince L, Loudon A. Clocking in to immunity. Nat Rev Immunol. 2018;18(7):423-437.

34. Allen NC, et al. Desynchronization of the molecular clock contributes to the heterogeneity of the inflammatory response. $S c i$ Signal. 2019;12(571):eaau1851.

35. Gibbs JE, et al. The nuclear receptor REV-ERB $\alpha$ mediates circadian regulation of innate immunity through selective regulation of inflammatory cytokines. Proc Natl Acad Sci U S A. 2012;109(2):582-587.

36. Gibbs J, et al. An epithelial circadian clock controls pulmonary inflammation and glucocorticoid action. Nat Med. 2014;20(8):919-926.

37. Zhang Z, et al. Genome-wide effect of pulmonary airway epithelial cell-specific Bmal1 deletion. FASEB J. 2019;33(5):6226-6238.

38. Kiessling S, Dubeau-Laramée G, Ohm H, Labrecque N, Olivier M, Cermakian N. The circadian clock in immune cells controls the magnitude of Leishmania parasite infection. Sci Rep. 2017;7(1):10892.

39. Pariollaud M, et al. Circadian clock component REV-ERB $\alpha$ controls homeostatic regulation of pulmonary inflammation. J Clin Invest. 2018;128(6):2281-2296.

40. Ryzhikov M, et al. Diurnal rhythms spatially and temporally organize autophagy. Cell Rep. 2019;26(7):1880-1892.e6.

41. Curtis AM, et al. Circadian control of innate immunity in macrophages by miR-155 targeting Bmal1. Proc Natl Acad Sci U S A. 2015;112(23):7231-7236.

42. Edgar RS, et al. Cell autonomous regulation of herpes and influenza virus infection by the circadian clock. Proc Natl Acad Sci U $S$ A. 2016;113(36):10085-10090.

43. Haspel JA, et al. Circadian rhythm reprogramming during lung inflammation. Nat Commun. 2014;5:4753.

44. Silver AC, Arjona A, Walker WE, Fikrig E. The circadian clock controls toll-like receptor 9-mediated innate and adaptive immunity. Immunity. 2012;36(2):251-261.

45. Heipertz EL, Harper J, Lopez CA, Fikrig E, Hughes ME, Walker WE. Circadian rhythms influence the severity of sepsis in mice via a TLR2-dependent, leukocyte-intrinsic mechanism. J Immunol. 2018;201(1):193-201.

46. Sengupta S, et al. Circadian control of lung inflammation in influenza infection. Nat Commun. 2019;10(1):4107.

47. Nguyen KD, Fentress SJ, Qiu Y, Yun K, Cox JS, Chawla A. Circadian gene Bmal1 regulates diurnal oscillations of Ly6C(hi) inflammatory monocytes. Science. 2013;341(6153):1483-1488.

48. Bellet MM, et al. Circadian clock regulates the host response to Salmonella. Proc Natl Acad Sci U S A. 2013;110(24):9897-9902.

49. Castanon-Cervantes O, et al. Dysregulation of inflammatory responses by chronic circadian disruption. J Immunol. 2010;185(10):5796-5805.

50. Oishi K, et al. Clock mutation affects circadian regulation of circulating blood cells. J Circadian Rhythms. 2006;4:13

51. Scheiermann C, et al. Adrenergic nerves govern circadian leukocyte recruitment to tissues. Immunity. 2012;37(2):290-301.

52. Zhao Y, et al. Uncoverin g the mystery of opposite circadian rhythms between mouse and human leukocytes in humanized mice. Blood. 2017;130(18):1995-2005.

53. Casanova-Acebes M, et al. Rhythmic modulation of the hematopoietic niche through neutrophil clearance. Cell. 2013;153(5):1025-1035.

54. Méndez-Ferrer S, Lucas D, Battista M, Frenette PS. Haematopoietic stem cell release is regulated by circadian oscillations. Nature. 2008;452(7186):442-447.

55. He W, et al. Circadian expression of migratory factors establishes lineage-specific signatures that guide the homing of leukocyte subsets to tissues. Immunity. 2018;49(6):1175-1190.e7. 
56. Druzd D, et al. Lymphocyte circadian clocks control lymph node trafficking and adaptive immune responses. Immunity. 2017;46(1):120-132.

57. A-Gonzalez N, et al. Phagocytosis imprints heterogeneity in tissue-resident macrophages. J Exp Med. 2017;214(5):1281-1296.

58. Suzuki K, Hayano Y, Nakai A, Furuta F, Noda M. Adrenergic control of the adaptive immune response by diurnal lymphocyte recirculation through lymph nodes. J Exp Med. 2016;213(12):2567-2574.

59. Wong SH, et al. Transcription factor ROR $\alpha$ is critical for nuocyte development. Nat Immunol. 2012;13(3):229-236.

60. Melo-Gonzalez F, Hepworth MR. Functional and phenotypic heterogeneity of group 3 innate lymphoid cells. Immunology. 2017;150(3):265-275.

61. Wu X, Tian J, Wang S. Insight into non-pathogenic Th17 cells in autoimmune diseases. Front Immunol. 2018;9:1112.

62. Eberl G, Marmon S, Sunshine MJ, Rennert PD, Choi Y, Littman DR. An essential function for the nuclear receptor RORgam$\mathrm{ma}(\mathrm{t})$ in the generation of fetal lymphoid tissue inducer cells. Nat Immunol. 2004;5(1):64-73.

63. Yang XO, et al. T helper 17 lineage differentiation is programmed by orphan nuclear receptors ROR alpha and ROR gamma. Immunity. 2008;28(1):29-39.

64. Yu X, et al. TH17 cell differentiation is regulated by the circadian clock. Science. 2013;342(6159):727-730

65. Amir M, et al. REV-ERB $\alpha$ regulates $\mathrm{T}_{\mathrm{H}} 17$ cell development and autoimmunity. Cell Rep. 2018;25(13):3733-3749.e8.

66. Carrillo-Vico A, Lardone PJ, Alvarez-Sánchez N, Rodríguez-Rodríguez A, Guerrero JM. Melatonin: buffering the immune system. Int J Mol Sci. 2013;14(4):8638-8683.

67. Farez MF, et al. Melatonin contributes to the seasonality of multiple sclerosis relapses. Cell. 2015;162(6):1338-1352.

68. Adrover JM, et al. A neutrophil timer coordinates immune defense and vascular protection. Immunity. 2019;50(2):390-402.e10.

69. Knauert MP, Haspel JA, Pisani MA. Sleep loss and circadian rhythm disruption in the intensive care unit. Clin Chest Med. 2015;36(3):419-429

70. Rechtschaffen A, Gilliland MA, Bergmann BM, Winter JB. Physiological correlates of prolonged sleep deprivation in rats. Science. 1983;221(4606):182-184.

71. Everson CA. Sustained sleep deprivation impairs host defense. Am J Physiol. 1993;265(5 pt 2):R1148-R1154.

72. Cappuccio FP, D'Elia L, Strazzullo P, Miller MA. Sleep duration and all-cause mortality: a systematic review and meta-analysis of prospective studies. Sleep. 2010;33(5):585-592.

73. Prather AA, Janicki-Deverts D, Hall MH, Cohen S. Behaviorally assessed sleep and susceptibility to the common cold. Sleep 2015;38(9):1353-1359.

74. Prather AA, et al. Sleep and antibody response to hepatitis B vaccination. Sleep. 2012;35(8):1063-1069.

75. Spiegel K, Sheridan JF, Van Cauter E. Effect of sleep deprivation on response to immunization. JAMA. 2002;288(12):1471-1472.

76. Benedict C, Brytting M, Markström A, Broman JE, Schiöth HB. Acute sleep deprivation has no lasting effects on the human antibody titer response following a novel influenza A H1N1 virus vaccination. BMC Immunol. 2012;13:1.

77. Brown R, Pang G, Husband AJ, King MG. Suppression of immunity to influenza virus infection in the respiratory tract following sleep disturbance. Reg Immunol. 1989;2(5):321-325.

78. Massie A, Boland E, Kapás L, Szentirmai É. Mice lacking alternatively activated (M2) macrophages show impairments in restorative sleep after sleep loss and in cold environment. Sci Rep. 2018;8(1):8625.

79. Ames C, Boland E, Szentirmai É. Effects of macrophage depletion on sleep in mice. PLoS ONE. 2016;11(7):e0159812.

80. Besedovsky L, Lange T, Haack M. The sleep-immune crosstalk in health and disease. Physiol Rev. 2019;99(3):1325-1380

81. Borbély AA, Daan S, Wirz-Justice A, Deboer T. The two-process model of sleep regulation: a reappraisal. J Sleep Res. 2016;25(2):131-143

82. Banerjee S, et al. Pharmacological targeting of the mammalian clock regulates sleep architecture and emotional behaviour. Nat Commun. 2014;5:5759.

83. Yu X, et al. Circadian factor BMAL1 in histaminergic neurons regulates sleep architecture. Curr Biol. 2014;24(23):2838-2844.

84. Laposky A, Easton A, Dugovic C, Walisser J, Bradfield C, Turek F. Deletion of the mammalian circadian clock gene BMAL1/ Mop3 alters baseline sleep architecture and the response to sleep deprivation. Sleep. 2005;28(4):395-409.

85. Möller-Levet CS, et al. Effects of insufficient sleep on circadian rhythmicity and expression amplitude of the human blood transcriptome. Proc Natl Acad Sci U S A. 2013;110(12):E1132-E1141.

86. Anafi RC, Pellegrino R, Shockley KR, Romer M, Tufik S, Pack AI. Sleep is not just for the brain: transcriptional responses to sleep in peripheral tissues. BMC Genomics. 2013;14:362.

87. Dijk DJ, Czeisler CA. Contribution of the circadian pacemaker and the sleep homeostat to sleep propensity, sleep structure, electroencephalographic slow waves, and sleep spindle activity in humans. J Neurosci. 1995;15(5 Pt 1):3526-3538.

88. Adams F. The extant works of Aretaeus the Cappadocian. London, United Kingdom: Sydenham Society; 1861.

89. Rijo-Ferreira F, et al. Sleeping sickness is a circadian disorder. Nat Commun. 2018;9(1):62.

90. Mullington JM, Simpson NS, Meier-Ewert HK, Haack M. Sleep loss and inflammation. Best Pract Res Clin Endocrinol Metab. 2010;24(5):775-784

91. Richardson MR, Churilla JR. Sleep duration and C-reactive protein in US adults. South Med J. 2017;110(4):314-317.

92. Ingram LA, Simpson RJ, Malone E, Florida-James GD. Sleep disruption and its effect on lymphocyte redeployment following an acute bout of exercise. Brain Behav Immun. 2015;47:100-108.

93. McAlpine CS, et al. Sleep modulates haematopoiesis and protects against atherosclerosis. Nature. 2019;566(7744):383-387.

94. Yang G, et al. Timing of expression of the core clock gene Bmall influences its effects on aging and survival. Sci Transl Med. 2016;8(324):324ra16.

95. Irwin M, Mascovich A, Gillin JC, Willoughby R, Pike J, Smith TL. Partial sleep deprivation reduces natural killer cell activity in humans. Psychosom Med. 1994;56(6):493-498.

96. Hemmers S, Rudensky AY. The cell-intrinsic circadian clock is dispensable for lymphocyte differentiation and function. Cell Rep. 2015;11(9):1339-1349.

97. Fortier EE, Rooney J, Dardente H, Hardy MP, Labrecque N, Cermakian N. Circadian variation of the response of T cells to antigen. J Immunol. 2011;187(12):6291-6300.

98. Long JE, Drayson MT, Taylor AE, Toellner KM, Lord JM, Phillips AC. Morning vaccination enhances antibody response over 
afternoon vaccination: a cluster-randomised trial. Vaccine. 2016;34(24):2679-2685

99. Lange T, Perras B, Fehm HL, Born J. Sleep enhances the human antibody response to hepatitis A vaccination. Psychosom Med. 2003;65(5):831-835.

100. Deng W, et al. The circadian clock controls immune checkpoint pathway in sepsis. Cell Rep. 2018;24(2):366-378.

101. Burris TP, Busby SA, Griffin PR. Targeting orphan nuclear receptors for treatment of metabolic diseases and autoimmunity. Chem Biol. 2012;19(1):51-59.

102. Cunningham PS, et al. Incidence of primary graft dysfunction after lung transplantation is altered by timing of allograft implantation. Thorax. 2019;74(4):413-416.

103. Vandergheynst A, Van de Borne P, Melot C, Preumont N, Knoop C, Leeman M. High prevalence of nocturnal arterial hypertension and non-dipping in lung transplant recipients. Acta Cardiol. 2010;65(4):395-400.

104. de Assis LVM, Kinker GS, Moraes MN, Markus RP, Fernandes PA, Castrucci AML. Expression of the circadian clock gene $B M A L 1$ positively correlates with antitumor immunity and patient survival in metastatic melanoma. Front Oncol. 2018;8:185

105. Mazzoccoli G, et al. Immune system alterations in lung cancer patients. Int J Immunopathol Pharmacol. 2003;16(2):167-174.

106. Yu H, Boyle TA, Zhou C, Rimm DL, Hirsch FR. PD-L1 expression in lung cancer. J Thorac Oncol. 2016;11(7):964-975.

107. Mizutani H, Tamagawa-Mineoka R, Minami Y, Yagita K, Katoh N. Constant light exposure impairs immune tolerance development in mice. J Dermatol Sci. 2017;86(1):63-70.

108. Jensen IJ, Sjaastad FV, Griffith TS, Badovinac VP. Sepsis-induced T cell immunoparalysis: the ins and outs of impaired T cell immunity. J Immunol. 2018;200(5):1543-1553.

109. Franceschi C, Garagnani P, Parini P, Giuliani C, Santoro A. Inflammaging: a new immune-metabolic viewpoint for age-related diseases. Nat Rev Endocrinol. 2018;14(10):576-590.

110. Mazzoccoli G, Carughi S, Sperandeo M, Pazienza V, Giuliani F, Greco A. Alteration of circadian rhythmicity of CD3+CD4+ lymphocyte subpopulation in healthy aging. J Biol Regul Homeost Agents. 2011;25(3):405-416.

111. O'Donnell AJ, Schneider P, McWatters HG, Reece SE. Fitness costs of disrupting circadian rhythms in malaria parasites. Proc Biol Sci. 2011;278(1717):2429-2436.

112. Mideo N, Reece SE, Smith AL, Metcalf CJ. The Cinderella syndrome: why do malaria-infected cells burst at midnight? Trends Parasitol. 2013;29(1):10-16.

113. Rijo-Ferreira F, Takahashi JS, Figueiredo LM. Circadian rhythms in parasites. PLoS Pathog. 2017;13(10):e1006590.

114. Hirako IC, et al. Daily rhythms of TNF $\alpha$ expression and food intake regulate synchrony of plasmodium stages with the host circadian cycle. Cell Host Microbe. 2018;23(6):796-808.e6.

115. Oetjen LK, et al. Sensory neurons co-opt classical immune signaling pathways to mediate chronic itch. Cell. 2017;171(1):217-228.e13

116. Trier AM, Kim BS. Cytokine modulation of atopic itch. Curr Opin Immunol. 2018;54:7-12.

117. Rosas-Ballina M, et al. Acetylcholine-synthesizing T cells relay neural signals in a vagus nerve circuit. Science. 2011;334(6052):98-101.

118. Reardon C, Murray K, Lomax AE. Neuroimmune communication in health and disease. Physiol Rev. 2018;98(4):2287-2316.

119. Patel T, Ishiuji Y, Yosipovitch G. Nocturnal itch: why do we itch at night? Acta Derm Venereol. 2007;87(4):295-298.

120. Griffin P, et al. Circadian clock protein Rev-erba regulates neuroinflammation. Proc Natl Acad Sci U S A. 2019;116(11):5102-5107.

121. Lananna BV, et al. Cell-autonomous regulation of astrocyte activation by the circadian clock protein BMAL1. Cell Rep. 2018;25(1):1-9.e5.

122. Musiek ES, Holtzman DM. Mechanisms linking circadian clocks, sleep, and neurodegeneration. Science. 2016;354(6315):1004-1008.

123. Tracey KJ. Physiology and immunology of the cholinergic antiinflammatory pathway. J Clin Invest. 2007;117(2):289-296.

124. Wang DW, Yin YM, Yao YM. Vagal modulation of the inflammatory response in sepsis. Int Rev Immunol. 2016;35(5):415-433.

125. Nobs SP, Tuganbaev T, Elinav E. Microbiome diurnal rhythmicity and its impact on host physiology and disease risk. $E M B O$ Rep. 2019;20(4):e47129.

126. McCoy KD, Ronchi F, Geuking MB. Host-microbiota interactions and adaptive immunity. Immunol Rev. 2017;279(1):63-69.

127. Leone V, et al. Effects of diurnal variation of gut microbes and high-fat feeding on host circadian clock function and metabolism. Cell Host Microbe. 2015;17(5):681-689.

128. Thaiss CA, et al. Transkingdom control of microbiota diurnal oscillations promotes metabolic homeostasis. Cell. 2014;159(3):514-529.

129. Zarrinpar A, Chaix A, Yooseph S, Panda S. Diet and feeding pattern affect the diurnal dynamics of the gut microbiome. Cell Metab. 2014;20(6):1006-1017.

130. Thaiss CA, et al. Microbiota diurnal rhythmicity programs host transcriptome oscillations. Cell. 2016;167(6):1495-1510.e12

131. Maury E, Ramsey KM, Bass J. Circadian rhythms and metabolic syndrome: from experimental genetics to human disease. Circ Res. 2010;106(3):447-462.

132. Sharma R, Sahota P, Thakkar MM. A single episode of binge alcohol drinking causes sleep disturbance, disrupts sleep homeostasis, and down-regulates equilibrative nucleoside transporter 1. J Neurochem. 2018;146(3):304-321.

133. Webb IC. Circadian rhythms and substance abuse: chronobiological considerations for the treatment of addiction. Curr Psychiatry Rep. 2017;19(2):12.

134. Casanova-Acebes M, et al. Neutrophils instruct homeostatic and pathological states in naive tissues. J Exp Med. 2018;215(11):2778-2795.

135. Toda H, Shi M, Williams JA, Sehgal A. Genetic mechanisms underlying sleep. Cold Spring Harb Symp Quant Biol. 2018;83:57-61.

136. Prendergast BJ, Cisse YM, Cable EJ, Zucker I. Dissociation of ultradian and circadian phenotypes in female and male Siberian hamsters. J Biol Rhythms. 2012;27(4):287-298.

137. West AC, Smith L, Ray DW, Loudon ASI, Brown TM, Bechtold DA. Misalignment with the external light environment drives metabolic and cardiac dysfunction. Nat Commun. 2017;8(1):417.

138. Ehlers A, et al. BMAL1 links the circadian clock to viral airway pathology and asthma phenotypes. Mucosal Immunol. 2018;11(1):97-111.

139. Hirota T, et al. Identification of small molecule activators of cryptochrome. Science. 2012;337(6098):1094-1097.

140. Resuehr D, Wu G, Johnson RL, Young ME, Hogenesch JB, Gamble KL. Shift work disrupts circadian regulation of the tran- 
scriptome in hospital nurses. J Biol Rhythms. 2019;34(2):167-177.

141. Anafi RC, Francey LJ, Hogenesch JB, Kim J. CYCLOPS reveals human transcriptional rhythms in health and disease. Proc Natl Acad Sci U S A. 2017;114(20):5312-5317.

142. Wittenbrink $\mathrm{N}$, et al. High-accuracy determination of internal circadian time from a single blood sample. JClin Invest. 2018;128(9):3826-3839.

143. Laing EE, Möller-Levet CS, Poh N, Santhi N, Archer SN, Dijk DJ. Blood transcriptome based biomarkers for human circadian phase. Elife. 2017;6:e20214.

144. Koshy A, Cuesta M, Boudreau P, Cermakian N, Boivin DB. Disruption of central and peripheral circadian clocks in police officers working at night. FASEB J. 2019;33(6):6789-6800.

145. Hughes ME, et al. Guidelines for genome-scale analysis of biological rhythms. J Biol Rhythms. 2017;32(5):380-393.

146. Paolino S, Cutolo M, Pizzorni C. Glucocorticoid management in rheumatoid arthritis: morning or night low dose? Reumatologia. 2017;55(4):189-197.

147. Matveyenko AV. Consideration for circadian physiology in rodent research. Physiology (Bethesda). 2018;33(4):250-251.

148. Sulli G, et al. Pharmacological activation of REV-ERBs is lethal in cancer and oncogene-induced senescence. Nature. 2018;553(7688):351-355.

149. Lamia KA, et al. AMPK regulates the circadian clock by cryptochrome phosphorylation and degradation. Science. 2009;326(5951):437-440.

150. Crosby P, et al. Insulin/IGF-1 drives PERIOD synthesis to entrain circadian rhythms with feeding time. Cell. 2019;177(4):896909.e20.

151. Miki T, Matsumoto T, Zhao Z, Lee CC. p53 regulates period2 expression and the circadian clock. Nat Commun. 2013;4:2444.

152. Belden WJ, Dunlap JC. SIRT1 is a circadian deacetylase for core clock components. Cell. 2008;134(2):212-214.

153. Honma S, et al. Dec1 and Dec2 are regulators of the mammalian molecular clock. Nature. 2002;419(6909):841-844.

154. Pick R, He W, Chen CS, Scheiermann C. Time-of-day-dependent trafficking and function of leukocyte subsets. Trends Immunol. 2019;40(6):524-537. 